\title{
METODOLOGÍA DE EXTENSIÓN PARA CREAR EMPRENDIMIENTOS PRODUCTIVOS: CASO DE ISLA VENADO, GOLFO DE NICOYA, COSTA RICA
}

\author{
METHODOLOGY OF EXTENSIVENESS TO GENERATE PRODUCTIVE ENTREPRENEURS: \\ THE CASE OF ISLA VENADO, GOLFO DE NICOYA, COSTA RICA
}

\section{Álvaro Martín Parada Gómez ${ }^{1}$}

\begin{abstract}
Resumen
Las empresas son uno de los cuatro componentes esenciales en el crecimiento de la producción y generación de ingresos de una economía. Las universidades contribuyen a través de proyectos de investigación y extensión al desarrollo de organizaciones sociales, cámaras, asociaciones de productores, lo cual conduce a la generación de empleo, producción e inversión. La función de extensión, entendida como vinculación de la universidad con los sectores sociales más vulnerables, implica crear oportunidades económicas. En este contexto, el propósito de este artículo es ofrecer una metodología para generar proyectos productivos que son potenciales micro o pequeñas empresas. Esta metodología es elaborada como parte del análisis de cadena productiva de la pesca artesanal en isla Venado, golfo de Nicoya de Costa Rica.
\end{abstract}

Palabras clave: desarrollo productivo; emprendimientos; metodología de extensión; vulnerabilidad social.

\begin{abstract}
Enterprises are one of the four essential components to increase production and generate income in an economy. Through research and outreach projects, universities contribute to the development of social
\end{abstract}

doi: http://dx.doi.org/10.15359/eys.20-48.2

Fecha de recepción: 14-05-2015. Fechas de reenvíos: 01-09-2015/05-10-15. Fecha de aceptación: 09-10-2015. Fecha de publicación: 02-11-15

${ }^{1} \mathrm{PhD}$ en Economía. Académico e investigador, Escuela de Economía, Universidad Nacional de Costa Rica. Correo electrónico: agomez@una.cr

Álvaro Martín Parada Gómez

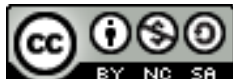

Revista Economía y Sociedad by Universidad Nacional is licensed under a Creative Commons Reconocimiento-NoComercial- 
organizations, chambers, and producers' associations, which results in employment, production, and investment. Outreach, understood as a link between the university and the most vulnerable social sectors, implies creating economic opportunities. In this context, the purpose of this article is to offer a methodology to generate productive projects that may become future micro o small businesses. This methodology is prepared as part of the analysis of the chain production of artisanal fisheries in Isla Venado, Gulf of Nicoya, Costa Rica.

Keywords: productive development; entrepreneurship; outreach methodology; social vulnerability.

\section{Introducción}

En el sistema económico las empresas son el componente esencial, comparativamente con las familias, el gobierno y los bancos. Estas son fundamentales para impulsar el crecimiento de la producción, la cual genera ingresos familiares que repercuten directamente en el bienestar de la sociedad (Black y Hartzenberg, 1997). Las universidades aportan al bienestar, a través del vínculo directo o indirecto con la sociedad, acciones que se concretizan en programas y proyectos de acción social y que son parte de la extensión universitaria. La vinculación entre la universidad y la sociedad, históricamente, se ha hecho a través de la extensión, entendida esta como una acción sustantiva que mejora las condiciones de vida de las familias y transforma la dinámica de las empresas, las cuales originan producción, empleo e inversión. Una de las formas de hacer extensión consiste en generar oportunidades económicas a través de proyectos socioproductivos que sustituyan las transferencias sociales tales como: bonos monetarios condicionadas a que las familias envíen a sus hijos a estudiar, como lo ha hecho en Costa Rica el Instituto Mixto de Ayuda Social (IMAS) con el programa Avancemos. De esta forma se han beneficiado: mujeres jefas de hogar, trabajadores por cuenta propia, jóvenes con bajo nivel de escolaridad, madres adolescentes, adulto mayor, entre otros. El marco institucional público se enfrenta a la disyuntiva de mantener el asistencialismo social o generar oportunidades económicas dentro de las cuales el emprendimiento productivo es una alternativa. En este contexto, el propósito de este artículo es sistematizar una metodología de extensión basada en la creación de emprendimientos para beneficiarios en condiciones de vulnerabilidad socioeconómica que rompen relativamente con el asistencialismo.

Este artículo se organiza de la siguiente forma: en la segunda parte se exponen elementos teóricos que orientan la metodología de emprendimientos productivos desarrollados en la localidad de isla Venado, golfo de Nicoya de Costa Rica. En la tercera, se explica la estructura metodológica definida para lograr formular y ejecutar los emprendimientos productivos. En la cuarta, se muestra la sistematización del caso de pesca artesanal en isla Venado desde la 
constitución de emprendimientos productivos. En la quinta y última sección se presentan algunas conclusiones.

\section{Elementos teóricos que orientan la metodología de emprendimientos productivos}

Los emprendimientos productivos son aquellos proyectos que son formulados y ejecutados de forma individual o colectiva. Dos condiciones deben cumplirse para emprender, primero es tener la "idea" para producir un bien o servicio que responde a una necesidad y segundo, ser capaz de llevarla a cabo. El empresario que piensa sin hacer tiene un accionar que se convierte en un conjunto vacío que no conduce a un resultado. Quien emprende es creativo porque constantemente está haciendo uso de la innovación para solucionar las dificultades que surgen en el contexto económico, social, político, cultural y ambiental en que se desarrolla. La persona que emprende asume riesgos y muestra pocos temores en los momentos propicios de asignación de recursos y factores productivos porque tiene seguridad del retorno por obtener dada la inversión realizada. La persona que emprende muestra fuerte pasión en cada actividad que realiza porque nunca renuncia a la consecución del objetivo trazado. Jofré al analizar aspectos modernos y gerenciales en las empresas señala que una persona emprendedora tiene muchas cualidades como: tolerancia al riesgo, trabajo en equipo, comunicación, respeto y compromiso, entre otros (Jofré, 1999).

Una interrogante que surge es la siguiente: ¿la persona emprendedora nace o se hace? Los dos aspectos están presentes en la dinámica de la realidad. Por un lado, el emprendedor puede hacerse con base en la formación y estudio de elementos teóricos y metodológicos relacionados con la empresariedad como por ejemplo la formulación de un proyecto, evaluación financiera, teoría de costos y producción, gestión estratégica, entre otros. Una vez asimilados este conjunto teórico y práctico, sin duda, la persona podrá emprender. Por otro lado, la persona puede nacer como emprendedora sin realizar mayores estudios o capacitación. Esto significa que alguien tiene una idea de proyecto productivo y a través de sus propias habilidades y destrezas logra formularlo, ejecutarlo y mantenerlo de manera sostenible a lo largo del tiempo. Al asumir ambas condiciones, se pueden definir algunos factores al éxito de los proyectos conducidos por la persona que emprende:

- Anteponer la humildad para aprender de otros, ante la soberbia, para confiar en el conocimiento innato.

- Anteponer la iniciativa para desarrollar ideas propias y no caer en la tentación de copiar y reproducir ideas de otros.

- Anteponer el trabajo permanente y tesonero para alcanzar los objetivos y combatir tanto la pereza, como falta de voluntad, lo cual limita el alcance de las metas.

- Anteponer la alta calidad y eficiencia en la elaboración de bienes y servicios, ante la producción basada en bajos costos y precios.

Álvaro Martín Parada Gómez

Revista Economía y Sociedad by Universidad Nacional is licensed under a Creative Commons Reconocimiento-NoComercial- 
- Anteponer el bien común como forma de bienestar colectivo, ante el bienestar individual que pregona la producción absoluta para el mercado.

- Anteponer la generación de bienes para nichos de mercado, ante aquella producción masiva de bienes para grandes mercados.

- Anteponer la apropiación de un mayor valor agregado a través de la integración vertical, ante la apropiación parcial y marginal en una actividad económica en particular.

El desarrollo de proyectos productivos se alimenta en parte de la base teórica que proviene de la economía neoclásica y clásica. Desde esta perspectiva, la producción de bienes es orientada estrictamente por el libre mercado y la acción individual de las personas en la sociedad (Smith, 1976). Quien emprende se enfrenta a mercados que soncompetencia perfecta, monopolios, oligopolios, entre otros. En estos mercados es mayoritariamente tomador de precios.

Los proyectos productivos se nutren de la base teórica de la producción, la empresa, los costos, la estructura de mercados, entre otros elementos. Lo anterior obliga a considerar componentes teóricos del análisis del sistema económico desde la perspectiva del comportamiento de la empresa, es decir, de la teoría microeconómica (Varian, 1994). También, otros autores como Pórter señalan que la competición de los mercados es necesaria para motivar las iniciativas emprendedoras, donde las empresas, estratégicamente, pueden lograr ventajas para competir a través de un proceso propio de creación y no por medio de la herencia de la dotación de los recursos naturales (Porter, 1990).

Los proyectos productivos logran generar autoempleo, inversión, producción y valor agregado y, fundamentalmente, cubren y satisfacen una o varias necesidades que la sociedad evidencia (Sapag, 2007). Cada proyecto es una empresa real o potencial que insume materias primas para hacer un proceso productivo que origina un producto final. Los factores productivos insumidos en la función de producción típicamente tradicional son el capital, el trabajo, la tierra y la tecnología (Varian, 1994). Desde esta perspectiva, la persona emprendedora hace la combinación de capital y trabajo aunado al uso de materias primas, lo cual lo potencia para alcanzar el resultado deseado.

Si el individuo que emprende produce, implica que genera ingresos que serán cuantificados según la cantidad de producción del bien $(\mathrm{k})$ que es vendida en los mercados al precio $\left(\mathrm{P}_{\mathrm{k}}\right)$, esto significa que hay ingresos que son formalizados a través de la función de ingreso total del bien $\mathrm{k}$, $I T_{k}=P_{k} . Q_{k}$. Lo sensato es que el proyecto genere ingresos brutos crecientes mayores a los costos a lo largo del ciclo de vida. Si esto es así, la empresa que se constituye logra estabilidad en la etapa inicial y puede potenciar un proceso de escalamiento en la producción en fases sucesivas a mediano y largo plazo. La estimación de los ingresos es relevante porque de estos depende la sostenibilidad financiera de la empresa. La condición de sostenibilidad depende del comportamiento de los precios, los cuales son típicamente definidos en los mercados de

4

Álvaro Martín Parada Gómez

(c) (i) (2)

Revista Economía y Sociedad by Universidad Nacional is licensed under a Creative Commons Reconocimiento-NoComercial- 
competencia perfecta o imperfecta. No obstante, la empresa pocas veces tiene influencia en el precio porque son dados por el mercado (Pindyck y Rubinfeld, 2001).

El proyecto productivo surge de una necesidad que origina la producción de un bien y servicio, por lo tanto, esa oferta del bien es creada por una empresa potencial. La empresa es una unidad de producción que realiza un conjunto de actividades económicas que originan un bien intermedio o final (Tirole, 1990). Esta producción requiere insumos que son parte de los factores productivos (capital, trabajo, tierra, tecnología). Este vector de factores productivos es definido a través de la función de costos, la cual se constituye en la restricción económica para alcanzar la posición óptima de beneficio. Se debe recordar que en el sistema económico los recursos son escasos y limitados. Por lo tanto, la definición del costo total del bien $\mathrm{k}\left(\mathrm{CT}_{\mathrm{k}}\right)$ al menos deberá partir de la siguiente función:

$$
\mathrm{CT}_{\mathrm{k}}=\mathrm{CVT}_{\mathrm{k}}+\mathrm{CFT}_{\mathrm{k}}
$$

Donde los costos variables $\left(\mathrm{CVT}_{\mathrm{k}}\right.$ ): se definen como aquellos costos que cambian al modificarse las unidades físicas de producción del bien $\mathrm{K}$ y los costos fijos ( $\mathrm{CFT}_{\mathrm{K}}$ ): Son aquellos que se mantienen constantes independiente de los distintos niveles de producción del bien K. Ambos, deben ser sistematizados para estimar la estructura de costos medios y relacionar estos con los ingresos.

Si asumimos que el único factor productivo variable es el trabajo, la empresa, desde la perspectiva de aporte y contribución de este a la producción, requerirá que este se forme y califique en relación con la función de producción. Por ejemplo: si la producción es pan será necesario calificar al panadero. Si se requiere producir queso será necesario preparar al quesero. El costo del recurso humano es un balance que debe elaborarse para poder determinar el costo de participación de este en el costo total. Desde el punto de vista del costo fijo, estos afectan marginalmente la producción y los costos fijos medios tienen una pendiente decreciente conforme el nivel de producción incrementa. La elaboración del balance de costos fijos incluiría al menos la electricidad, el agua, la telefonía, así como la depreciación de activos fijos como maquinaria, equipo, vehículos, entre otros.

El principio de sostenibilidad económica de la empresa es un criterio irrenunciable si se quiere sobrevivir en los mercados y ser un emprendedor exitoso. Por lo tanto, la relación entre ingresos y costos debe ser positiva, en donde se cumpla la siguiente función de beneficio para el bien $\mathrm{k}: \mathrm{IT}_{\mathrm{k}}-\mathrm{CT}_{\mathrm{k}}=\mathrm{B}_{\mathrm{k}}$

La función de beneficio, absolutamente, determina el beneficio bruto $\left(B_{k}\right)$, por lo que es muy usual observar proyectos socioeconómicos con beneficios negativos o nulos debido a la mala estructuración de costos e ingresos. Si los beneficios son nulos y la empresa logra cubrir los costos variables medios, se justifica socialmente la continuidad del proyecto o empresa en el mercado porque al menos está generando empleo que permite ingresos suficientes para la manutención de

Álvaro Martín Parada Gómez 
la familia, esto si el proyecto es propio. Desde la perspectiva de economía social, Razeto ha indicado que el crédito y los préstamos son esenciales para generar oportunidades económicas (proyectos productivos). No obstante, afirma que las empresas nuevas y pequeñas nunca tienen un gran patrimonio y enfrentan siempre tasas de interés elevadas comparativamente con la tasa de beneficio obtenida (Razeto, 2014). Si esto ocurre con seguridad el efecto en el bienestar será limitado. También, al investigarse el sector textil de Costa Rica en el año 2005 determinó que el crédito es uno de los factores que debe ser superado por las empresas pequeñas debido al exceso de garantías que exigen los bancos (Parada, 2012).

El emprendedor que lidera una empresa pequeña opera en mercados que en teoría tienden a la competencia perfecta. No obstante, los mercados, de forma permanente, son distorsionados por fallas de mercado conducentes a un alto poder de mercado y con concentraciones de empresas. Las imperfecciones más evidentes son las economías de escala, la integración vertical, información asimétrica, entre otros. El resultado es estructuras de mercado oligopólicas o monopólicas que atentan contra el bienestar de la sociedad (Ferguson y Ferguson, 1994).

El producto o servicio ofrecido por la persona emprendedora no puede obviar las condiciones en los mercados, estos, teóricamente, muestran relación con la cadena productiva en la que opera la empresa o la unidad productiva. La cadena es una estructura constituida por eslabones que inician con el diseño del producto o servicio, apropiación de materias primas, en donde se pasa por el proceso productivo, avanza hacia el mercadeo y la comercialización hasta que el consumidor final adquiere el bien o servicio. Si esto es así, quien emprende requiere conocer el comportamiento de los cuatro mercados que median en estas cadenas por razones económicas y de sostenibilidad.

El primer mercado es el de insumos o materias primas necesarias para elaborar el bien final. En este las materias primas y bienes auxiliares son adquiridos para luego ser transformados. El precio, el costo y las cantidades son las variables determinantes para optimizar las decisiones en este eslabón.

El segundo mercado es el de productos finales donde la empresa ofrece lo que produce y los consumidores adquieren los bienes en función de las infinitas necesidades. En este caso la variación de los precios es el determinante porque de estos depende la estabilidad de los ingresos reales. Sobre el control de precios los bancos centrales hacen esfuerzos por estabilizarlos y mantener el poder adquisitivo de los agentes económicos que intervienen en el sistema económico. Por lo tanto, el buen manejo de los precios en los mercados es esencial para la pequeña empresa (Massad y Patillo, 2000). Adicionalmente, para aquellas empresas que producen bienes exportables y otras que importan requieren interpretar los efectos del tipo de cambio, aranceles y barreras no arancelarias que tienen afectaciones sobre las empresas que compiten en mercados internacionales.

6

Álvaro Martín Parada Gómez

(c) (i) (2) (2)

Revista Economía y Sociedad by Universidad Nacional is licensed under a Creative Commons Reconocimiento-NoComercial- 
El tercer mercado es el de trabajo, el cual está relacionado con la determinación de los costos variables y uso del factor trabajo. Si se hace abstracción de la calidad de este, el costo del trabajo será determinado por el salario. Por lo tanto, la orientación de la política de gobierno y/o de Estado en la definición de la tasa de salarios afecta la funcionalidad de la empresa conducida por el emprendedor. Los empresarios no tienen margen de determinación de la tasa salarial, no obstante hacen presión a la baja a través de las cámaras y gremios existentes, quienes intentan permanentemente negociar con el gobierno.

El cuarto mercado es el financiero en el cual se determina la tasa de interés. La tasa de interés es pasiva y activa. La primera es la que recibe el ahorrante por ceder los recursos financieros a los bancos para que sean prestados. La segunda, es la que paga el individuo al comportarse como inversor, es decir, asume el costo financiero de la inversión. En ambos casos, la tasa de interés es dada y el productor o emprendedor debe aceptarla. En este caso es determinante que el empresario pueda analizar de manera permanente la dinámica de la tasa de interés, así como la política crediticia que definan los hacedores de política macroeconómica del gobierno. Los elementos del entorno económico nacional e internacional no siempre son estudiados o analizados por el empresariado, y se enfrenta muchas veces adversidades y amenazas que causan quiebras o inestabilidades a corto plazo en las empresas.

En este contexto del mercado se posicionan quienes emprenden y las empresas. La dinámica de cada mercado puede significar oportunidades y amenazas que deben ser enfrentadas con creatividad para mantenerse exitosamente en los mercados. Una amenaza permanente es la presencia de la intermediación en la comercialización que implica mayor apropiación de beneficios en este eslabón de la cadena productiva comparativamente con los pequeños productores. Por ejemplo, en el mercado de tomate "bola" en Costa Rica, el productor vendió en finca a 0,58 centavos de dólar, en el mercado mayorista de Cenada a 0,81 centavos, y 1,19 dólares en el mercado Borbón y el propietario del supermercado paga el precio de 2,38 dólares y vende al consumidor final a 4,0 dólares en la cadena de supermercados (Cenada, 2014). El diferencial en los precios es la intermediación que establece cada actor que participa en la comercialización.

El intermediario "compra barato y vende caro". Normalmente, el productor hace la función de producción bastante bien, pero no logra posicionarse en la comercialización por razón de desconocimiento de los mercados y actitudes positivas para vender los productos. Ante esta condición, el comercializador surge como una alternativa para el productor, quien en la mayoría de los casos no hace mayor esfuerzo por avanzar hacia adelante en la cadena y poder apropiarse del beneficio tasado y generado por la acción de intermediar. Por lo tanto, el desarrollo de habilidades y destrezas del productor y o emprendedor en la comercialización es un determinante para el escalamiento en el intervalo del ciclo de vida del proyecto productivo y empresa.

Álvaro Martín Parada Gómez

(c) (i) (-)

Revista Economía y Sociedad by Universidad Nacional is licensed under a Creative Commons Reconocimiento-NoComercial- 


\section{Estructura metodológica para la generación de emprendimientos productivos}

La metodología para generar emprendimientos está influenciada por la forma de estructurar un proyecto productivo. Se asume que un proyecto productivo origina una empresa que entra a competir en mercados donde los consumidores adquieren los bienes y/o servicios producidos 0 elaborados. Esta metodología es un proceso que integra la formación de grupos de beneficiarios que son permanentemente capacitados en tiempos predeterminados de forma continua. La formulación de estos proyectos en el marco de un proceso de capacitación y generación de capacidades implica seguir el horizonte del ciclo de vida de un proyecto. La metodología general para impulsar la generación de emprendimientos socioeconómicos está definida estratégicamente de acuerdo con las siguientes fases:

\section{A. Determinar el cuadro o perfil de vulnerabilidad}

Tabla 1.

Perfil de beneficiarios según variables por indicadores. 2014.

\begin{tabular}{|c|c|c|}
\hline VARIABLES & DEFINICIÓN & INDICADOR \\
\hline Educación & $\begin{array}{l}\text { Se refiere al grado académico cursado } \\
\text { formalmente }\end{array}$ & $\begin{array}{l}\text { \% de la población con formación } \\
\text { formal }\end{array}$ \\
\hline Salud & $\begin{array}{l}\text { Pertenece al régimen de invalidez, } \\
\text { vejez y muerte }\end{array}$ & $\begin{array}{l}\% \text { de la población con cobertura } \\
\text { social (C.C.S.S.) }\end{array}$ \\
\hline Ingreso & $\begin{array}{l}\text { Ingreso recibido formal } \\
\text { informalmente }\end{array}$ & Ingreso promedio mensual \\
\hline Empleo & $\begin{array}{l}\text { Disponibilidad de trabajo...total o } \\
\text { parcial }\end{array}$ & \% de ocupación \\
\hline Género & $\begin{array}{l}\text { Integración de la mujer a las } \\
\text { actividades económicas }\end{array}$ & $\begin{array}{l}\% \text { de mujeres con actividades } \\
\text { económicas en marcha }\end{array}$ \\
\hline Vivienda & Disponibilidad de vivienda & \% población con vivienda propia \\
\hline Familia & Número de miembros de la familia & $\begin{array}{l}\text { Número promedio de integrantes en } \\
\text { la familia }\end{array}$ \\
\hline
\end{tabular}

Fuente: elaboración propia.

El perfil del beneficiarios es necesario para explicar el nivel de desarrollo humano de aquellos que forman empresas y emprendedores. Metodológicamente, es recomendable establecer criterios asociados con las condiciones de vivienda, infraestructura, género, ingresos, entre otras. De esta manera, las distintas variables mostradas son parte del perfil por elaborar.

8 


\section{B. Determinar el cuadro o perfil de empresariedad}

La empresariedad es el proceso de formación de la empresa a través de las áreas esenciales relacionadas con el crecimiento empresarial, tales como: costos, producción, administración y gestión, mercadeo y comercialización, entre otras. Metodológicamente, es recomendable elaborar un perfil del beneficiario considerando algunas variables de la empresariedad que generen información para definir un proceso de formación de capacidades empresariales de acuerdo con los requerimientos de los beneficiarios

\section{Tabla 2.}

Perfil de beneficiarios según variables por indicadores. 2014.

\begin{tabular}{lll}
\hline \multicolumn{1}{c}{ VARIABLES } & \multicolumn{1}{c}{ DEFINICIÓN } & \multicolumn{1}{c}{ INDICADOR } \\
\hline Actividad económica & $\begin{array}{l}\text { Se refiere a la actividad que origina } \\
\text { el producto intermedio o final }\end{array}$ & $\begin{array}{l}\text { Tipo de actividad: \% agrícola, \% } \\
\text { industrial, \% comercio y servicios }\end{array}$ \\
$\begin{array}{l}\text { Experiencia } \\
\text { Productos }\end{array}$ & $\begin{array}{l}\text { Años de creación de la empresa } \\
\text { Son los productos elaborados }\end{array}$ & $\begin{array}{l}\text { Número de años de existencia } \\
\text { Número de productos elaborados } \\
\text { Producción media y total }\end{array}$ \\
Ingresos & Ingresos por ventas & $\begin{array}{l}\text { Ingreso total } \\
\text { Costos variable total } \\
\text { Costos }\end{array}$ \\
Son los costos de producción & $\begin{array}{l}\text { Costos fijo total } \\
\text { Costo total y costo medio }\end{array}$ \\
Precio & Precio de los productos & $\begin{array}{l}\text { Precio nominal } \\
\text { Empresa }\end{array}$ \\
Inversión & Tamaño del proyecto o empresa & Metrados en infraestructura \\
Cantidad de dinero disponible para & Monto de inversión en colones \\
Mercado & el proyecto productivo & \% mercado local, regional, nacional e \\
& Participación de mercado & internacional
\end{tabular}

Fuente: elaboración propia. Nota aclaratoria: pueden ser incluidas más variables relacionadas con la teoría de la empresa, costos, producción y mercados.

\section{Organizar la cadena productiva del bien o servicio según eslabón}

La organización de la cadena productiva del bien o servicio que se producirá permite comprender la dinámica del proyecto o empresa dentro de un proceso integral y contexto de mercados. Algunos autores afirman que "cadena productiva se refiere al ámbito rango de actividades involucradas en el diseño, producción y comercialización de un producto donde la constitución de los conglomerados es importante para el desarrollo productivo" (Gereffi Bair, 2001). Para organizar la cadena productiva al menos deben entenderse los principales elementos: 
a) Describir la apropiación de insumos y cuantificar costos de factores productivos.

b) Explicar el proceso productivo y cuantificar costos, producción, diseño y proceso productivo.

c) Explicar el proceso de mercadeo y comercialización considerando el margen de intermediación en la comercialización.

d) Detallar el consumo final del bien elaborado.

La cadena productiva en su forma general integra los elementos antes mencionados. Esta se muestra en la figura 1.

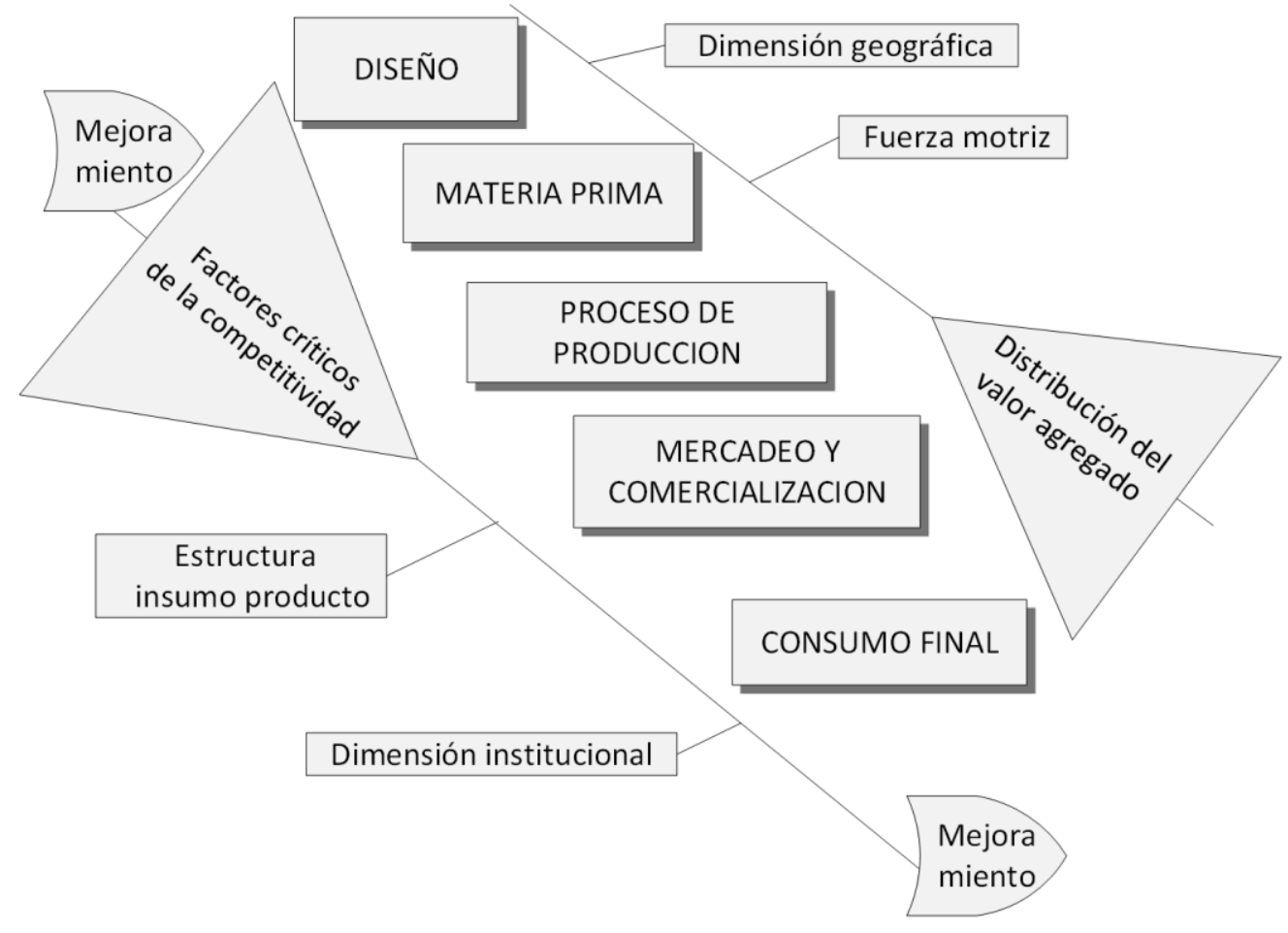

Figura 1. La cadena global de mercancías en su forma general. Fuente: Parada 2011, figura 3.1, página 68.

La cadena global de valor está integrada por una serie de eslabones que inicia con el diseño, la apropiación de materias primas, el proceso productivo, el mercadeo y comercialización y concluye con el consumo final. Estos eslabones son amplias redes de trabajo que generan valor en el sistema económico. La idea de utilizar el enfoque de la cadena en la orientación de los procesos de producción es detectar y evidenciar los factores críticos que afectan la

10 
productividad y eficiencia de los proyectos productivos. Esto permite introducir mejoras continuas en el recurso humano, tecnología, procesos productivos, entre otros.

La base indicativa de la cadena productiva permite hacer un balance de a, b, c, metodológico, para poder definir un programa de capacitación conducente a la elaboración del proyecto productivo y/o empresa. Típicamente, la planificación de la formación está en función del balance de requerimientos. La conclusión ha sido que el proceso de empresariedad debe iniciarse de acuerdo con las necesidades. En la tabla 3 se muestra un conjunto de necesidades conducentes a tener áreas de formación en los distintos beneficiarios.

Tabla 3.

Necesidades de los beneficiarios según área de formación. 2014.

\begin{tabular}{ll}
\hline \multicolumn{1}{c}{ NECESIDAD DE FORMACIÓN } & \multicolumn{1}{c}{ ÁREA DE FORMACIÓN } \\
\hline Establecimiento de costos de producción & Estructuración de costos \\
Organización de la producción & Producción(diseño y procesos) \\
Organización de la empresa y/o proyecto & Gestión estratégica \\
Definición de la oportunidad económica & Formulación y evaluación del proyecto de inversión \\
Conocimiento del mercado & Mercadeo y comercialización \\
Estabilidad y desarrollo en el ambiente de trabajo & Liderazgo y clima organizacional \\
\hline
\end{tabular}

Fuente: elaboración propia. Nota aclaratoria: pueden ser incluidas más variables relacionadas con la teoría de la empresa, costos, producción y mercados. Esta tabla solo explica el vector de empresariedad como fuente de creación de los proyectos y empresas.

\section{Elementos operativos de la metodología}

La creación de capacidades para generar un proyecto productivo conducente a conformar una empresa requiere utilizar un conjunto de instrumentos y medios para organizar y sistematizar el proceso de empresariedad. Entre estas acciones e instrumentos se mencionan los siguientes:

a) Realización de entrevistas a los beneficiarios.

b) Aplicación de cuestionarios (perfiles).

c) Análisis de $a \mathrm{y} b$.

d) Elaboración de módulos de capacitación según necesidades de empresariedad.

e) Aplicación de los módulos de empresariedad a través de talleres dirigidos por el especialista (extensionista).

f) Elaboración de proyectos productivos en conjunto con los beneficiarios.

Álvaro Martín Parada Gómez

Revista Economía y Sociedad by Universidad Nacional is licensed under a Creative Commons Reconocimiento-NoComercial- 
g) Articular el financiamiento de los proyectos productivos con entidades bancarias en el sistema financiero.

h) Actividades de seguimiento de los proyectos a través de visitas a beneficiarios, reuniones informales con productores, talleres de retroalimentación.

La aplicación de los módulos ha sido conceptualizada bajo el enfoque de ciclo de vida de una empresa o proyecto. La formación continua de los beneficiarios es esencial en procesos de empresariedad, donde se considera la continuidad para garantizar el éxito en el marco del crecimiento de la curva de aprendizaje relacionada con el proyecto productivo o empresa por crear (Parada, 2007).

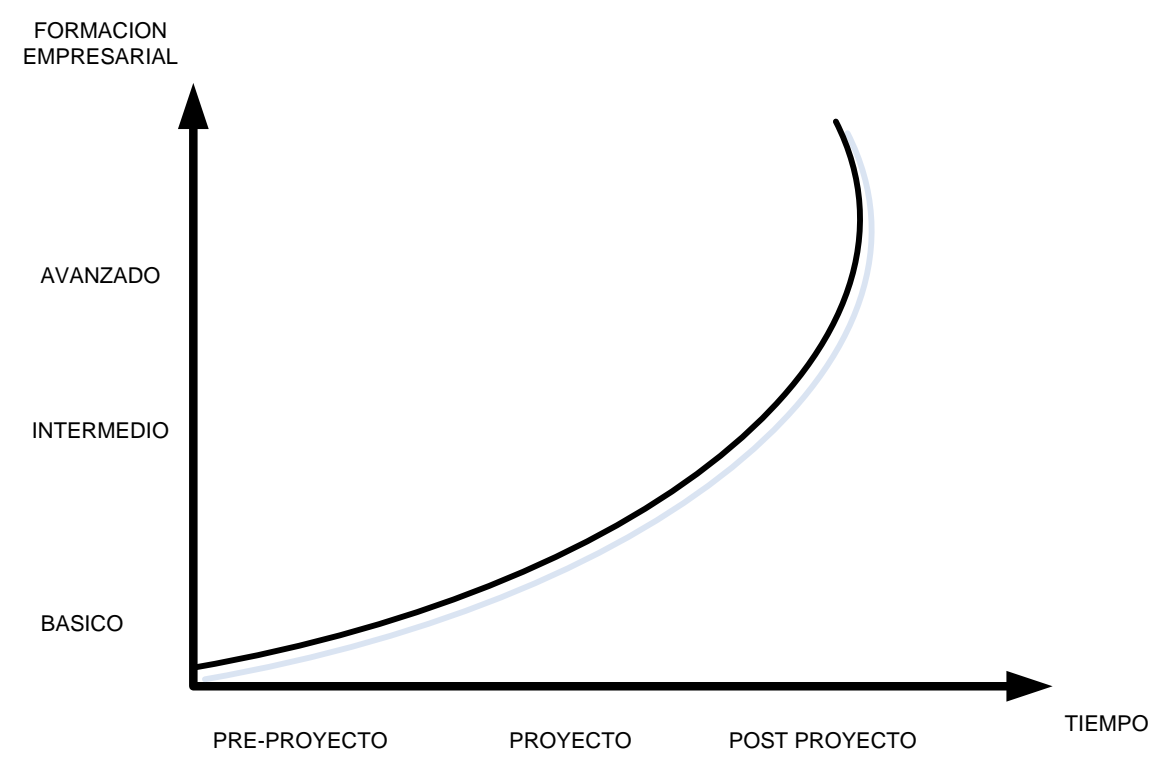

Figura 2. Fases de la formación empresarial. Fuente: elaboración propia según aporte al proyecto Focco Mipyme. Escuela de Economía. UNA. 2007.

La figura 2 muestra la curva de aprendizaje por inducir a través de la formación continua, la cual debe evolucionar conforme el proyecto productivo se desenvuelve. Las fases de formación contemplan niveles de enseñanza-aprendizaje básicos que evolucionan en el tiempo hacia los niveles intermedios y avanzados dentro de una concepción de proceso continuo en el horizonte de crecimiento del proyecto. Si esto es así, en el inicio el preproyecto debe ser elaborado con el apoyo técnico basado en la formulación de un proyecto. Una vez formulado el proyecto, se pone en marcha y en paralelo se requiere crear capacidades empresariales en un nivel intermedio en áreas esenciales como costos, mercadeo y comercialización. En un tercer momento, en el proceso de madurez del proyecto la experiencia y el aprendizaje previo permiten avanzar en la formación continua

12

Álvaro Martín Parada Gómez

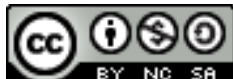

Revista Economía y Sociedad by Universidad Nacional is licensed under a Creative Commons Reconocimiento-NoComercial- 
hacia un nivel de capacitación mayor (avanzado). Adicionalmente, la asesoría técnica y el seguimiento es un requerimiento para poder garantizar que la producción del bien será un éxito. Desde la temporalidad del ciclo de vida de un proyecto productivo se estima que se requieren tres años para estabilizar el proyecto, lo cual es el embrión de una micro o pequeña empresa. La empresa evoluciona a partir del año tres y continúa su crecimiento y desarrollo.

El proceso metodológico explicado anteriormente ha permitido generar proyectos productivos sostenibles financieramente desde la Universidad Nacional de Costa Rica (UNA) en la localidad de isla Venado en el golfo de Nicoya, Costa Rica. Prácticamente, los componentes teóricos y metodológicos fueron abordados para crear un supermercado pequeño, una pequeña fábrica de hielo y reforzar la gestión empresarial de una pescadería. Estos proyectos productivos fueron desarrollados en el marco de apoyo y soporte del proyecto Focco Mipyme ejecutado en la Escuela de Economía (UNA). Esta experiencia es sistematizada en el siguiente apartado.

\section{Sistematización de la experiencia: caso isla Venado, golfo de Nicoya, Costa Rica}

\section{Caso de pesca artesanal isla Venado}

La isla Venado está ubicada en la zona del Pacífico Norte de Costa Rica, específicamente en el golfo de Nicoya, forma parte de un conjunto de islas: isla Venado, isla Caballo e isla Bejuco, las cuales pertenecen al cantón central (Lepanto), provincia de Puntarenas; es una isla pequeña, cuenta con una extensión de 3,5 kilómetros cuadrados y la población es de aproximadamente 1.300 habitantes. Las carencias sociales profundizan la pobreza de las familias que habitan en isla Venado. En inicios del siglo veintiuno, la falta de vivienda, agua potable, adecuada atención de salud, el alto desempleo, ausencia de oportunidades económicas, entre otras, son deficiencias en el desarrollo local que limita el bienestar social de las familias que mayoritariamente se dedican a la pesca artesanal.

Isla Venado es una localidad organizada, en su mayoría, por pescadores que históricamente han hecho de la pesca artesanal una fuente de ingresos familiares. El análisis de caso de esta isla se hace con el propósito de evidenciar la formulación y ejecución de proyectos socioproductivos impulsados por emprendedores en condiciones de vulnerabilidad social y económica. 


\section{Caracterización de isla Venado}

Tabla 4.

Características generales de isla Venado 2010.

\begin{tabular}{ll}
\hline \multicolumn{1}{c}{ VARIABLES } & \multicolumn{1}{c}{ CARACTERÍSTICAS } \\
\hline LARIABLES GEOGRÁFICAS \\
Localización & $\begin{array}{l}\text { Zona del Pacífico Norte del golfo de Nicoya. Cantón central de } \\
\text { Lepanto. Puntarenas. Es parte del complejo de islas que } \\
\text { conforman el golfo, tales como isla Caballo, Bejuco y San Lucas. }\end{array}$ \\
Extensión geográfica & 3,5 kilómetros cuadrados \\
Población & 1.300 habitantes \\
\hline & VARIABLES ECONÓMICAS \\
\hline Actividad económica principal & Pesca artesanal \\
Ingreso mensual & 350 dólares \\
Empleo & 6 de cada 10 \\
\hline & VARIABLES SOCIALES \\
\hline Familias en pobreza & 6 de cada 10 \\
Viviendas deficientes & 5 de cada 10 \\
Hogares con carencias & 5 de cada 10 \\
\hline
\end{tabular}

Fuente: entrevista aplicada a pescadores y observación de la actividad productiva (2010). Informe parcial proyecto: Pesca Artesanal en Isla Venado, Golfo de Nicoya. Escuela de Economía. 2012.

Isla Venado es una comunidad que económicamente depende de la pesca artesanal y socialmente muestra serios problemas como la pobreza y el desempleo. La falta de oportunidades económicas, debido a la ausencia de inversionistas y de proyectos productivos y la escasa generación de fuentes de empleo, han hecho que los habitantes no alcancen el bienestar socioeconómico que se experimenta en las ciudades urbanas del Valle Central como San José, Heredia y Alajuela. La alta dependencia de la pesca artesanal ha vuelto vulnerables a las familias porque en época de auge se observa aumento en los ingresos, pero en períodos de poca dotación del recurso pesquero los ingresos son mínimos y/o nulos.

La pobreza es observable en las familias debido a la falta de ingresos para cubrir necesidades básicas como: salud, educación, vivienda, alimentación, vestido y calzado, es decir, no alcanzan a recibir el salario mínimo establecido por el Ministerio de Trabajo de Costa Rica (2015), fijado en salario mínimo ubicado en 525,5 USD. La alimentación se centra mucho en el consumo de mariscos porque no se dispone de dinero para adquirir frutas, carnes rojas y blancas, leche, huevo, entre otros bienes. Las familias más antiguas de la isla son las que han podido consolidar la propiedad de tierras y viviendas dignas, el pescador tradicional habita en casas improvisadas que carecen de los servicios básicos como electricidad y agua potable. La población joven asiste

14

Álvaro Martín Parada Gómez

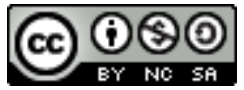

Revista Economía y Sociedad by Universidad Nacional is licensed under a Creative Commons Reconocimiento-NoComercial- 
a la educación primaria y secundaria, pero son pocos los graduados que continúan estudios en la educación superior y la gran mayoría termina sumándose a la pesca artesanal o busca opciones de trabajo en la zona que normalmente son de baja remuneración. La actividad económica no ha desarrollado los servicios, el comercio es limitado y los procesos industriales o agroindustriales son incipientes, lo cual ha hecho vulnerable el tejido productivo.

\section{Organización general de la producción de pesca artesanal (Cadena productiva)}

La cadena de pesca artesanal en Isla Venado está compuesta por el eslabón de apropiación de materias primas y auxiliares que se han dividido en capital fijo y circulante. El fijo se refiere al conjunto de equipo y maquinaria que se requiere para realizar la actividad productiva $y$ experimenta desgaste en el proceso de producción (depreciación). El capital circulante es el conjunto de materias auxiliares o primas que son consumidas parcialmente o totalmente en el proceso de producción. Si se asume esta definición, se puede indicar que el hielo para preservar el pescado y el agua empleada para lavarlo son partes del capital circulante. Las neveras para preservar el pescado en el centro de acopio utilizan electricidad y normalmente tienen un máximo de 250 kilogramos de capacidad. La panga es el medio de transporte estratégico para realizar la captura del pescado en mar abierto, la cual se mueve por motores que insumen gasolina y aceite como aditivo. En la actividad de la pesca son utilizados bienes auxiliares como lámparas de luz para alumbrar la panga por la noche, el aceite que es mezclado con la gasolina en el motor de la panga, entre otros. Todos los insumos son esenciales para iniciar la captura de pesca en mar abierto en la isla.

La captura del pescado, camarón, entre otras especies se realiza en mar abierto durante el tiempo de pesca de la disponibilidad (oferta) de mariscos en general en la zona del golfo de Nicoya. La experiencia del pescador le indica en donde están los bancos de peces y con base en la localización de estos se trasladan para instalar las redes conocidas como trasmallos que normalmente miden 350 metros; no obstante, algunos pescadores utilizan trasmallos que tiene un máximo de 850 metros y hasta 1.000 metros. Los pescadores salen todos los días; en una salida de pesca normalmente en un día se capturan 100 kilogramos de pescado, pero en días de abundancia se capturan hasta 400 kilogramos por día (Parada, 2012). El centro de acopio paga 2 USD dólares por kilogramo, es decir, después de una salida donde ocurra el máximo de pesca el pescador obtendría 800 dólares de ingresos en un día. Por lo tanto, la pesca genera ingresos familiares que, administrados adecuadamente, permitirían un nivel de bienestar satisfactorio. 


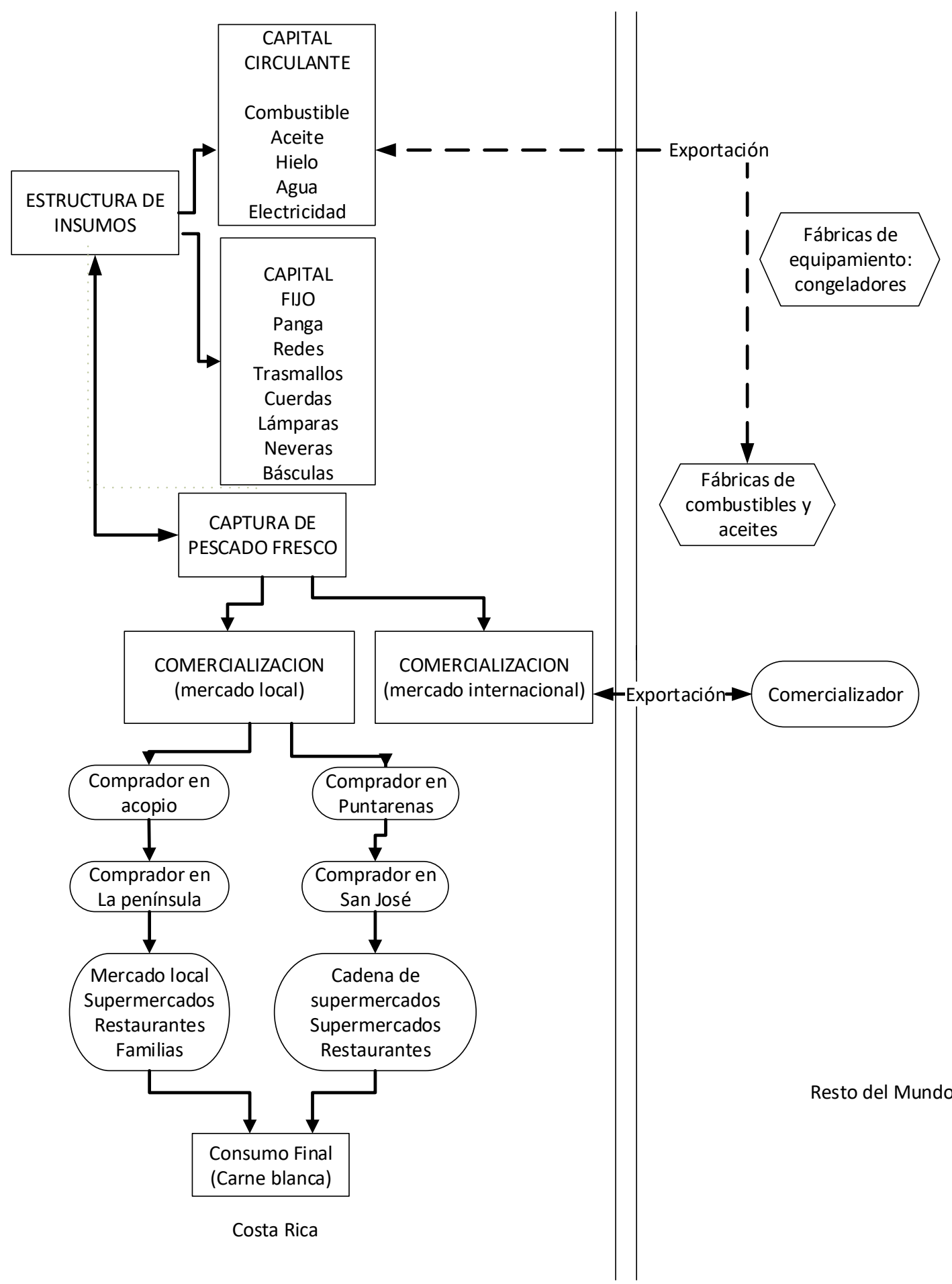

Figura 3. La cadena de pesca artesanal Isla Venado, Golfo de Nicoya. 2012. Fuente: elaboración propia con base en información de pescadores de Isla Venado 2012.

16

Álvaro Martín Parada Gómez

(c) (i)(2)

Revista Economía y Sociedad by Universidad Nacional is licensed under a Creative Commons Reconocimiento-NoComercial- 
La comercialización del pescado inicia una vez que el pescador lleva la cantidad de captura al centro de acopio. El centro de acopio recibe comercializadores que ingresan a la isla en busca de pescado fresco para comercializarlo posteriormente. Dos opciones se observaron, posteriores al depósito en el centro de acopio. La primera es la venta que hace el centro de acopio al comercializador que ingresa a la isla, quien compra el pescado en el sitio y lo comercializa en la península de Nicoya. La segunda, es la venta que el centro de acopio hace a comercializadores en Puntarenas. Estos adquieren el pescado fresco y lo venden a comercializadores que buscan pescado en Puntarenas y lo comercializan en mercados de la Gran Área Metropolitana (GAM). En la GAM el comercializador distribuye el pescado en cadenas de supermercados, supermercados independientes, restaurantes especializados y mercados populares. En este eslabón se percibe la presencia de muchos comercializadores que cobran sobreprecios, producto de la labor que realizan. En esta amplia red de comercialización el pescador está atrapado y se convierte en aceptante del precio fijado por los comercializadores.

El consumo final del pescado es realizado por consumidores independientes que adquieren el pescado en distintos puntos de comercialización como lo son supermercados y mercados populares. También, las familias y consumidores independientes asisten a restaurantes especializados en mariscos para consumir el pescado preparado en platillos como por ejemplo: pescado entero, filete de pescado, ceviche, entre otros. En realidad, los restaurantes marcan el patrón de consumo de acuerdo con las preferencias de los consumidores finales. Lo que aún no se tiene cuantificado en esta relación de comercialización es cuánto valor agregado es generado y apropiado por cada comercializador, interrogante que será importante clarificar en investigaciones posteriores.

\section{Alternativas productivas en la dinámica de la cadena de pesca artesanal}

Las alternativas productivas en la pesca artesanal se relacionan con la conformación de la cadena productiva. En el eslabón de materia prima debe indicarse que la apropiación del hielo es determinante de la calidad del producto. En primer lugar, se justifica la creación de una pequeña fábrica de hielo. Este proyecto, implica formulación y evaluación como un requisito para determinar la factibilidad económica. Adicionalmente, capacitación y asesoría técnica para que los pescadores líderes realizaran el proyecto. La capacitación se fundamentó en los costos y la producción del hielo para afianzar la formación empresarial. El aprendizaje fue recíproco entre los pescadores y académicos porque el intercambio de trabajo en los talleres significó entender las necesidades de los pescadores y así hacer la adecuación respectiva en materia de los contenidos con respecto a la formulación y evaluación de proyectos. Los resultados económicos del proyecto fueron positivos y se generó información para la adecuada toma de decisiones.

La segunda alternativa consistió en la "creación de un supermercadito". La idea surge producto de la necesidad de comercializar bienes de primera necesidad que son demandados por los pobladores de isla Venado. La interrogante por resolver fue la siguiente: ¿por qué los pescadores quieren incursionar en la comercialización? Inmediatamente, había que entender 
que en el eslabón de la comercialización siempre el beneficio es mayor que en la producción y esto representaba una oportunidad de diversificación de la actividad económica para los pescadores. Por lo tanto, en el corto plazo los pescadores organizados fueron orientados hacia la formulación del proyecto y su evaluación. Ellos entendieron los elementos fundamentales como los costos de operación, la infraestructura requerida, la gestión para el manejo de las ventas, entre otros elementos. Finalmente, el proyecto fue valorado económicamente y entró en operación en el año 2010, para el 2014 era un proyecto productivo estable con beneficios positivos para los pescadores que, humildemente, ahora cuentan con una pescadería, una pequeña fábrica de hielo y un supermercadito.

La organización de la cadena productiva evidencia actividades económicas que tienen potencial de desarrollo y contribución en la comunidad. Adicionalmente, la iniciativa emprendedora de los pobladores en la localidad ha mostrado el surgimiento de actividades como la elaboración de artesanías y el turismo.

\section{La capacitación y asesoría técnica a las alternativas productivas (pesca)}

La organización de la cadena productiva de pesca artesanal donde se desarrollan múltiples proyectos productivos que dinamizan la actividad evidencian factores relacionados con la gestión empresarial de los pescadores que deben ser mejorada para que la actividad económica sea exitosa. Lo relevante de definir la cadena es que ha permitido determinar los factores por modificar tales como: la estructuración de costos, el mercadeo y la comercialización, la planificación empresarial, entre otros. La capacitación en primera instancia es un mecanismo útil para crear capacidades y corregir deficiencias de gestión de las pequeñas empresas. Adicionalmente, la asesoría técnica refuerza la gestión empresarial y el buen funcionamiento de la empresa. En el caso de un proyecto como la fábrica de hielo y la pescadería reflejan la necesidad de las capacitaciones para garantizar la sostenibilidad de la pequeña empresa.

\section{El marco institucional de apoyo a la actividad}

El marco institucional del país ha pretendido impulsar y generar el soporte adecuado a la localidad y a la actividad económica a través de las siguientes funciones:

- Gestión de apoyo socioeconómico para pescadores artesanales.

- Investigación y transferencia tecnológica.

- Modernización institucional.

- Organizaciones de productores.

- Ordenar el acceso y el aprovechamiento de los recursos pesqueros y acuícolas.

- Promover la comercialización e industrialización de los recursos pesqueros acuícolas.

18

Álvaro Martín Parada Gómez

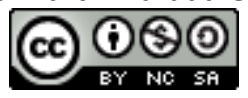

Revista Economía y Sociedad by Universidad Nacional is licensed under a Creative Commons Reconocimiento-NoComercial- 
Estas funciones han sido operacionalizadas a través de más de una decena de instituciones que han mostrado asistencia en las que sobresalen, según función, las siguientes (ver tabla 5):

\section{Tabla 5.}

Instituciones de apoyo a la actividad de pesca en isla Venado según función

\begin{tabular}{|c|c|}
\hline INSTITUCIÓN & FUNCIÓN \\
\hline 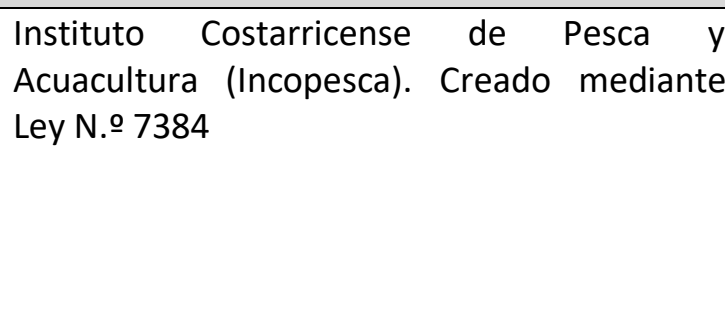 & $\begin{array}{l}\text { Promover, ordenar y coordinar el sector pesquero y de } \\
\text { acuicultura, con el propósito de lograr la conservación, } \\
\text { el aprovechamiento y el uso sostenible de los recursos } \\
\text { biológicos del mar y de la acuicultura, así como dar } \\
\text { seguimiento y aplicación a la legislación para el } \\
\text { desarrollo de la pesca, la caza marítima y evitar la } \\
\text { contaminación de los recursos marítimos y acuícolas }\end{array}$ \\
\hline Instituto Mixto de Ayuda Social (IMAS) & $\begin{array}{l}\text { Desarrollo de programas para ciudadanos en condición } \\
\text { de pobreza (en este caso pescadores) }\end{array}$ \\
\hline Ministerio de Agricultura y Ganadería & $\begin{array}{l}\text { Introducción de especies foráneas destinadas para el } \\
\text { cultivo (otorga los permisos) }\end{array}$ \\
\hline
\end{tabular}

Fuente: Planes estratégicos de cada institución. 2012. Nota aclaratoria: el marco institucional de apoyo se extiende a muchas instituciones llamadas por sus siglas INA, MARVIVA, MIDEPLAN, MEIC, entre otras que no han sido incluidas en el detalle.

Las instituciones realizan esfuerzos para apoyar el desarrollo de isla Venado a través de la actividad pesquera. No obstante, la pesca tiene límites naturales de reproducción continua del recurso en el área geográfica del golfo de Nicoya, lo cual hace más difícil la respuesta a las necesidades sociales y vulnerabilidades de las familias pesqueras. Las funciones son consistentes para cada institución, pero el resultado esperado es difícil de alcanzar debido a la burocracia institucional que no permite responder en tiempo adecuado a las demandas de los pescadores. En el país en el año 2012 se confirmó que para realizar los trámites para abrir una pequeña empresa se requerían de 60 días (Banco Mundial: Doing Bussiness Report, 2012). También, la falta de recursos financieros y humanos debido a los limitados presupuestos públicos, entre otros factores. Sin embargo, la presencia institucional está evidenciada en la localidad y aporta al desarrollo de las familias pesqueras desde sus potencialidades y capacidades.

Con seguridad son muchas las oportunidades económicas que existen en isla Venado, pero se requiere de emprendedores más que instituciones públicas, que tomen las decisiones en materia de progreso y desarrollo socioeconómico en la comunidad. 


\section{Conclusiones}

Las personas emprendedoras nacen y/o se hacen. Las que nacen tienen habilidades y destrezas como la pasión por crear y producir bienes bajo sus propias iniciativas. Aquellos que se hacen tienen actitudes hacia un aprendizaje técnico y académico conducente a hacer realidad las ideas y transformarlas en proyectos productivos y empresas, es decir, tienen la capacidad de pensar y hacer. En el sistema económico surgen ambos y compiten por tener una posición exitosa en los mercados. El hacer emprendedores implica formación y creación de capacidades en el campo empresarial (empresariedad). Se requiere desarrollar destrezas y habilidades en el manejo de los costos de la empresa, el mercadeo y comercialización para tener mejor posición en el mercado, entre otras más. Por lo tanto, la formación continua ayuda a que el empresario (a) avance exitosamente en el mercado doméstico.

La sostenibilidad económica de los proyectos productivos es necesaria mantener beneficios positivos que conduzcan al crecimiento escalonado de la producción en el marco del ciclo de vida del proyecto socioproductivo o empresa. La sostenibilidad en los proyectos ayuda a reducir las demandas sociales por transferencias directas o indirectas de aquellas instituciones que asumen la función de soporte a la producción.

La población de beneficiarios al tener condiciones de vulnerabilidad, tales como estar en niveles de pobreza, bajo nivel de escolaridad, desempleo, madres adolescentes, adulto mayor, entre otros, implica enfrentar obstáculos y adversidades en el proceso de creación de capacidades en ámbitos como, por ejemplo: apropiación de capacidades en empresariedad, uso y adopción tecnológica, manejo de estrategias de mercado, gestión estratégica, entre otros componentes; los cuales son necesarios para constituir de forma sostenible una empresa o proyecto productivo con carácter de mediano y largo plazo.

La acción social genera un proceso de aprendizaje muy peculiar que se "vive" en el campo y no en las aulas universitarias. Es en la realidad donde a través de un método de trabajo estructurado se interactúa con la población beneficiaria. Este método no es absolutamente perfecto porque en la mayoría de los casos se deben hacer modificaciones de contenidos, formas de enseñar, formas de aprender y comunicar. Los beneficiarios tienen conocimiento que es comunicado y debe ser aprendido por parte del extensionista, resistirse a comprenderlo podría ser peligroso para la consecución de los objetivos comunes. Por lo tanto, la acción social es en doble vía y conveniente. Además, la humildad es una herramienta fundamental para el ejercicio, puesto que se deben valorar, anteponer y utilizar los conocimientos de los actores cercanos a la realidad que se quiere mejorar.

Los proyectos socioproductivos que producen bienes y servicios de distinta índole requieren valorar el comportamiento de las variables de los precios, puesto que afectan desde lo externo la sostenibilidad productiva, tales como: los salarios, los precios de los bienes, la tasa de interés 20

Álvaro Martín Parada Gómez

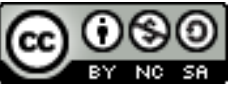

Revista Economía y Sociedad by Universidad Nacional is licensed under a Creative Commons Reconocimiento-NoComercial- 
y el tipo de cambio. La integración de estas es esencial para la toma de buenas decisiones en las empresas productivas. La toma de decisiones por parte de los propietarios de las empresas o sus gestores en muchos casos ignoran estos comportamientos y asumen, innecesariamente, afectaciones en la tasa de beneficio producto de las variaciones en las variables precio que alteran o incrementan los costos de producción, de tal manera que encarecen los productos y reducen la capacidad de competir de las empresas en el mercado.

\section{Referencias}

Black, P., Hartzenberg, T., Standish, B. (1997). Economics principles \& practice (Second Edition).London: PITMAN PUBLISHIN.

Banco Mundial. (2012). Doing Business Report (Informe). Recuperado de http://espanol.doingbusiness.org/reports/global-reports/doing-business-2012

Ferguson, P., Ferguson, G. (1994). Industrial Economics: Issues and perspectives (Second edition).

Recuperado

de https://books.google.co.uk/books/about/Industrial Economics.html?id=LqcndL hTP4C

Gereffi, G., J, Bair. (2001). Local Clusters in Global Chains: The Causes and Consequences of Export Dynamism Tin Terreon's Blue Jeans Industry. WorldDevelopment, 29(11), 18851903. http://dx.doi.org/10.1016/S0305-750X(01)00075-4

Gereffi, G., M,Korzeniewicz.(1994). Commodity Chain and Global Capitalism. Recuperado de https://books.google.co.cr/books?id=A86j9pWfTcAC\&redir esc=y

Jofré, A. (1999). Enfoques gerenciales modernos (Segunda Edición). MARS Editores:San José, Costa Rica.

Massad, C., Patillo, C. (2000). Macroeconomía en un mundo interdependiente. Recuperado de https://books.google.co.cr/books/about/Macroeconom\%C3\%ADa en un mundo inte rdependie.html?id=EMk4AAAACAAJ\&redir esc=y

Ministerio de Trabajo y Seguridad Social de Costa Rica. (5 de Diciembre de 2014). Fijación de salarios mínimos para el sector privado que regirán a partir del 1 de enero del 2015(Decreto Ejecutivo No.38728).La Gaceta No. 235. Recuperado de http://www.gaceta.go.cr/pub/2014/12/05/COMP 0512 2014.pdf 
Parada, M. (2007). Proyecto de formación continua de las capacidades competitivas de las Mipymes de Costa Rica (Informe final). Heredia: Universidad Nacional de Costa Rica.

Parada, M. (2011). Presente y futuro de la industria de ropa en Costa Rica: Un enfoque de cadena de mercancías (Tesis de Doctorado,Tilburg University). Recuperada de https://pure.uvt.nl/portal/en/publications/presente-y-futuro-de-la-industria-de-ropaen-costa-rica(c49888f9-bfe0-4f00-aa78-33ded770464d).html

Parada, M. (2012). Análisis de la pesca artesanal en Isla Venado (Informe final).Heredia: Universidad Nacional de Costa Rica.

Pindyck, R., Rubinfeld, D. (2001). Microeconomía (Quinta Edición). Recuperado de https://books.google.ca/books?id=F3FkQgAACAAJ\&hl=es\&source=gbs book other ver sions

Porter, M (1990). The competitive advantage of nations.http://dx.doi.org/10.1002/cir.3880010112

Razeto, L. (2014). Creación de empresas asociativas y solidarias: Curso teórico-práctico. Recuperado de https://books.google.co.cr/books/about/Creaci\%C3\%B3n_de empresas asociativas_y soli.html?id=v7cknwEACAAJ\&redir esc $=y$

Sapag, N. (2007). Proyectos de inversión formulación y evaluación. Recuperado de https://books.google.co.cr/books?id=pIS1QnFYt5IC\&redir esc=y

Smith, A. (1976).An Inquiry into the Nature and Causes of the Wealth of Nations. The University of Chicago Press.Recuperado de http://press.uchicago.edu/ucp/books/book/chicago///bo3637045.html

Tirole, J. (1990). La teoría de la Organización Industrial. Recuperado de https://books.google.com.pe/books/about/La teor\%C3\%ADa de la organizaci\%C3\%B 3n industria.html?id=X5HcPQAACAAJ

Varian H. (1994). Microeconomía Intermedia: Un Enfoque Moderno (Tercera Edición). Recuperado de https://books.google.co.cr/books/about/Microeconom\%C3\%ADa intermedia.html?id= Yw1OIAAACAAJ\&redir esc $=y$

22 